\title{
Progression of Clinical Features in Lewy Body Dementia Can Be Detected Over 6 Months
}

Elie Matar, MD, Simon R. White, PhD, John-Paul Taylor, MD, PhD, Alan Thomas, MD, PhD, Ian G. McKeith, MD, FMedSci, Joseph P.M. Kane, PhD, Ajenthan Surendranathan, MD, Glenda M. Halliday, PhD, Simon J.G. Lewis, MD, PhD, and John T. O'Brien, DM, FRCPsych

Neurology ${ }^{\circledR}$ 2021;97:e1031-e1040. doi:10.1212/WNL.0000000000012450

\section{Abstract}

\section{Objective}

This study aimed to quantify the trajectory and magnitude of change of the key clinical features and corresponding symptom domains of dementia with Lewy bodies (DLB) and Parkinson disease dementia (PDD), including global cognition, parkinsonism, recurrent visual hallucinations, cognitive fluctuations, and sleep disturbance.

\section{Methods}

One hundred sixteen patients with Lewy body dementia (DLB $=72$, PDD $=44$ ) underwent assessment at baseline and 3 and 6 months as part of a prospective multicenter randomized controlled trial. Linear mixed models were constructed for core outcome measures using the Mini-Mental State Examination (MMSE), motor section of the Unified Parkinson's Disease Rating Scale (UPDRS-III), Dementia Cognitive Fluctuations Scale (DCFS), and Neuropsychiatric Inventory (NPI).

\section{Results}

Within the time frame of our study (6 months), we were able to identify a significant cognitive decline of 1.3 points on the MMSE $(p=0.002)$ and significant worsening of motor parkinsonism with an increase in UPDRS-III score of 3.2 points $(p=0.018)$. Fluctuation severity also increased using the DCFS with a 6 -month change in score of 1.3 points $(p=0.001)$. Uniquely, a signal for increased severity of sleep symptoms of 1.2 points (NPI-sleep) was also detectable $(p$ $=0.04$ ). Significant changes in neuropsychiatric symptoms were not detected. There was no difference in rates of change of scores between DLB and PDD.

\section{Discussion}

Clinically significant rates of change in core clinical features can be detected and quantified in Lewy body dementia over a relatively short period (6 months) using common clinical instruments and thus may be useful as clinical endpoints for therapeutic trials of diseasemodifying and symptomatic agents.

\author{
Correspondence \\ Dr. Matar \\ elie.matar@sydney.edu.au
}

\section{MORE ONLINE}

(ค) Podcast

Dr. Jeff Ratliff talks to

Dr. Elie Matar about the paper "Progression of Clinical Features in Lewy Body Dementia Can Be Detected Over 6 Months." NPub.org/4tw4uj

From the Department of Psychiatry (E.M., S.R.W., A.S., J.T.O.) and MRC Biostatistics Unit (S.R.W.), University of Cambridge, UK; Forefront Parkinson's Disease Research Clinic (E.M., G.M.H., S.J.G.L.) and Brain and Mind Centre (E.M., G.M.H., S.J.G.L.), Faculty of Medicine and Health, University of Sydney, Australia; Newcastle Translational and Clinical Research Institute (J.-P.T., A.T., I.G.M.), Campus for Ageing and Vitality, Newcastle University, Newcastle Upon Tyne; and Centre for Public Health (J.P.M.K.), Queen's University Belfast, UK.

Go to Neurology.org/N for full disclosures. Funding information and disclosures deemed relevant by the authors, if any, are provided at the end of the article. 


\section{Glossary}

$\mathrm{AD}=$ Alzheimer disease $\mathbf{C I}=$ confidence interval DCFS = Dementia Cognitive Fluctuations Scale DIAMOND = Diagnosis and Management of Neurodegenerative Dementia; DLB = dementia with Lewy bodies; MMSE = Mini-Mental State Examination; MoCA = Montreal Cognitive Assessment; NHS = National Health Service; NPI = Neuropsychiatric Inventory; PDD = Parkinson disease dementia; UPDRS = Unified Parkinson's Disease Rating Scale.

Dementia with Lewy bodies (DLB) and Parkinson disease dementia (PDD), referred to collectively as Lewy body dementia, are characterized by the presence of dementia accompanied by shared clinical features, including motor parkinsonism, recurrent visual hallucinations, cognitive fluctuations, and REM sleep behavior disorder. ${ }^{1-3}$

Despite the significant public health impact, ${ }^{4-7}$ major challenges in the field have resulted in a relative paucity of symptomatic and disease-modifying trials in DLB and PDD. ${ }^{8}$ One such challenge is the delineation of appropriate outcome measures for tracking disease progression and severity. Indeed, few prospective studies have directly investigated the natural history of key clinical features in Lewy body dementia. ${ }^{6,9-13}$ Existing studies have focused mainly on changes in cognitive outcomes $^{9-11,14-16}$ with little known about the natural history of neuropsychiatric features, ${ }^{17}$ motor parkinsonism, fluctuations, and sleep symptoms, especially over the 6-month time frames typical of clinical trials. ${ }^{18,19}$ Such information would be critical for the incorporation of these features as endpoints in future clinical trials.

To address this gap, we sought to quantify the trajectory and magnitude of change of the key clinical features and symptom domains of cognitive impairment, motor parkinsonism, cognitive fluctuations, and neuropsychiatric and sleep disturbances in a cohort of patients with PDD and DLB over a period of 6 months using widely used clinical rating scales. We analyzed data from a prospective multicenter nonpharmacologic intervention trial. ${ }^{20}$ The nature of the intervention, designed to encourage expected standards of care, made it ideal for assessing the natural progression of clinical features under the conditions of a clinical trial.

\section{Methods}

\section{Participants}

Data from 127 participants with Lewy body dementia (77 DLB, 50 PDD) were analyzed from a cluster randomized trial (Diagnosis and Management of Neurodegenerative Dementia [DIAMOND-Lewy] Study; ISRCTN11083027) ${ }^{20}$ performed between 2016 and 2017 in 23 memory or movement disorder services across 8 main jurisdictions (trusts) of the UK National Health Service (NHS; 4 trusts in North East England and 4 trusts in East Anglia). All patients underwent clinical assessment and were diagnosed as having probable DLB or PDD according to current consensus criteria. ${ }^{2,3}$ Half of the services were randomized to receive a management toolkit comprising a summary of current evidence-based guidelines ${ }^{21}$ for symptomatic treatment of Lewy body dementia (made freely available since the conclusion of the study), while the other services continued with standard care (control arm). From these, 131 participants were recruited to be assessed at baseline and 3 and 6 months. Of those recruited, 127 participants underwent baseline assessments (Figure 1). Because this study assessed the rate of change, data for at least 2 time points were required to contribute to the group-level trajectories. Therefore, patients who could not be followed up after the baseline measurement $(\mathrm{n}=$ 11) were excluded, leaving 116 participants ( 72 DLB, 44 PDD) for the final analysis (including 107 patients with data for all 3 time points, 7 patients with data at baseline and 3 months, and 2 patients with data at baseline and 6 months). Results of the trial with respect to the main intervention have recently been published. ${ }^{20}$

\section{Standard Protocol Approvals, Registrations, and Patient Consents}

Ethics approval for this study was obtained from the NHS Research Ethics Committee. Written informed consent was obtained from all participants and their caregivers/next of kin.

\section{Clinical Variables}

All baseline and 3- and 6-month assessments were conducted by the same research team members in each respective region (North East England and East Anglia, UK) blinded to the service allocation (toolkit or standard care). Core clinical features were assessed with commonly used and validated research instruments. Cognition was measured using the Montreal Cognitive Assessment (MoCA) and the MMSE. Motor parkinsonism was graded by use of Section III of the Movement Disorder Society Unified Parkinson's Disease Rating Scale (UPDRS-III). ${ }^{22}$ Cognitive fluctuations were assessed with the Dementia Cognitive Fluctuation Scale (DCFS) ${ }^{23}$ Neuropsychiatric symptoms were assessed with the Neuropsychiatric Inventory (NPI). ${ }^{24}$ The NPI assesses 12 behavioral domains according to an interview with the caregiver. To maximize the ability to detect a rate of change in this variable, the trajectories of neuropsychiatric symptoms were analyzed for 3 predetermined scores derived from the NPI: the total NPI score summed across all 12 items; the NPI hallucinations score (NPI-hallucinations; frequency $\times$ severity); and a 4-item subscore (NPI-4) calculated as the sum of scores for 4 items of hallucinations, delusions, depression, and apathy. ${ }^{25}$ The NPI-4 has been identified in a previous study to consist of items sensitive to Lewy body dementia and has been used as a primary efficacy endpoint in previous randomized 
Figure 1 Consolidated Standards of Reporting Trials Diagram Including Enrollment, Registration, and Rates of Completion of Study

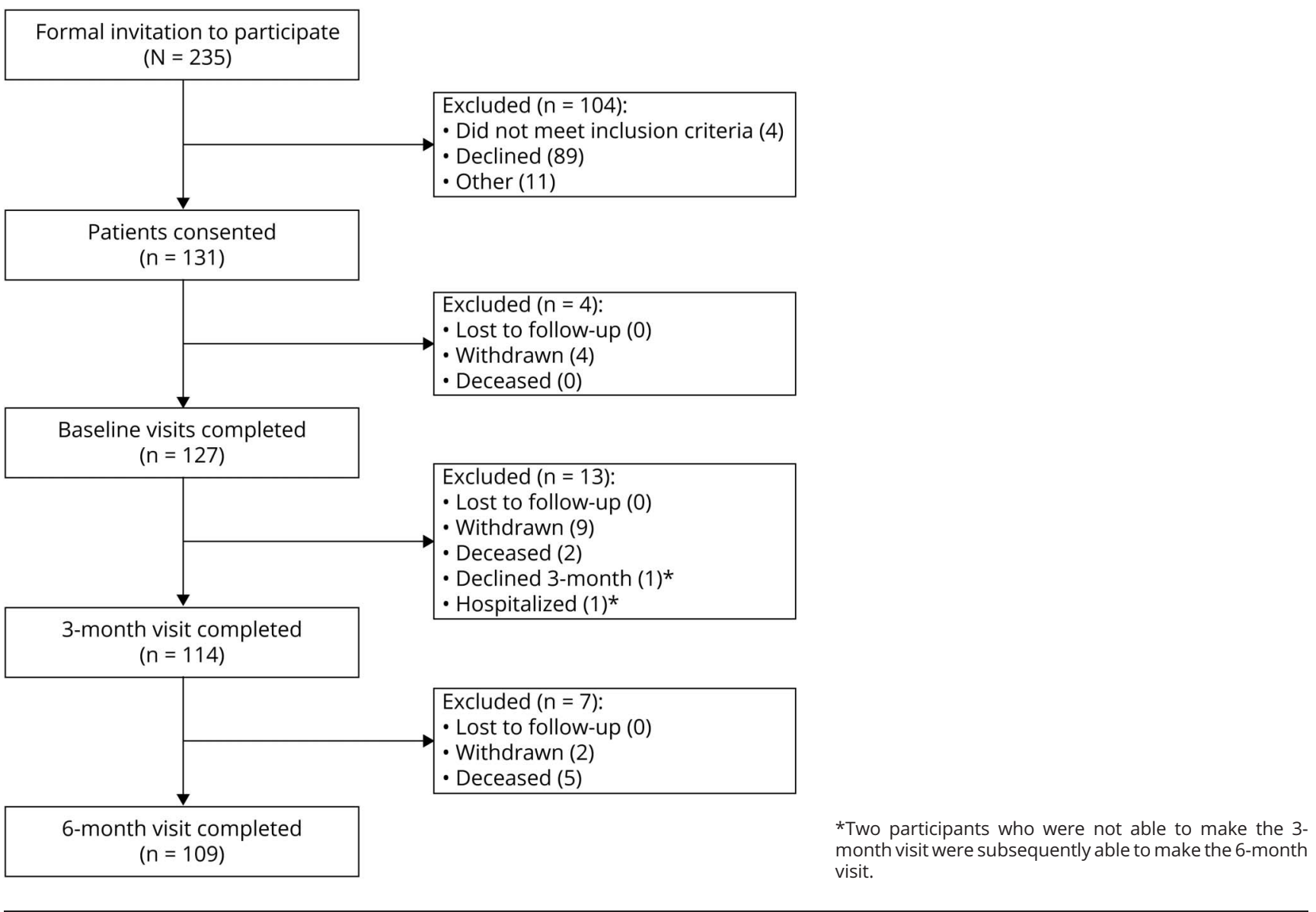

trials. ${ }^{19,26}$ Sleep disturbance was also analyzed with the sleep subscore of the NPI (NPI-sleep) as a patient-centered and clinically relevant indicator of sleep quality.

All medications and their dosages were recorded at each visit. Over the course of the trial, 3 patients $(2.6 \% ; 3$ control, 0 intervention) had changes in the dose/type of antipsychotic medication, 20 patients (17.2\%; 8 control, 12 intervention) had changes in dopaminergic medication, and 21 patients (18.1\%; 13 control, 8 intervention) had changes in acetylcholinesterase inhibitors.

\section{Statistical Analysis}

All statistical analyses were performed with the IBM Statistical Package for the Social Sciences (version 26.0.0, IBM Corp, Armonk, NY). Descriptive statistics (regarding baseline) are presented as mean (SD) or frequency (percentage). While accounted for in the mixed modeling below, baseline comparison between DLB and PDD with respect to variables of interest was performed with a 2-tailed independent-samples $t$ test or $\chi^{2}$ test as appropriate $(\alpha$ level $=0.05)$.

To explore longitudinal changes of the variables of interest, we applied linear mixed-effects modeling using time as a fixed effect and intercept and slope as random effects. This statistical approach was felt to be best suited to account for the natural heterogeneity in the severity of features at baseline and individual variation in longitudinal trajectories. ${ }^{27}$ The outcomes of interest (dependent variables) included the scores derived from the MMSE, MoCA, UPDRS-III, DCFS, NPI (total), NPI-4, NPI-hallucinations, and NPI-sleep. A linear mixed-effect model was constructed for each outcome of interest. An individualized set of prespecified fixed effects (in addition to time) were included in each model constituting confounding variables pertaining to the outcome of interest (specified in the Results section). Although there was no significant difference between intervention and control groups at baseline or follow-up in any of the above outcomes of interest, the binarized intervention status (control vs management toolkit) was included as a fixed effect in all models to account for any potential contribution. Because the primary hypothesis was that we would expect longitudinal progression in the variables of interest, time (in months) was included as a continuous, fixed effect in all models with time 0 occurring from the date of baseline measurement. Random intercepts and slopes were grouped by individual and fitted with a normal distribution around a zero mean. The Wald test was used to test for the significance of random effects in the 
Table 1 Baseline Characteristics

\begin{tabular}{|c|c|c|c|}
\hline Characteristic & DLB + PDD $(n=116)$ & DLB $(n=72)$ & $\operatorname{PDD}(n=44)$ \\
\hline Age, y & $77.9 \pm 7.2$ & $77.1 \pm 7.1$ & $79.2 \pm 6.9$ \\
\hline Male, n (\%) & $91(78.4)$ & $55(76.4)$ & $36(81.2)$ \\
\hline Intervention group, $\mathrm{n}$ (\%) & $63(54.3)$ & $42(58.3)$ & $21(47.7)$ \\
\hline MMSE score & $21.5 \pm 5.9$ & $21.7 \pm 5.9$ & $20.5 \pm 6.6$ \\
\hline MoCA score & $15.7 \pm 5.4$ & $15.8 \pm 5.4$ & $14.9 \pm 5.7$ \\
\hline Parkinsonism, $\mathrm{n}(\%)^{\mathbf{b}}$ & $87(75)$ & $52(72.2)$ & $44(100)$ \\
\hline Visual hallucinations, n (\%) & $82(70.7)$ & $44(61.1)$ & $37(84.1)$ \\
\hline Fluctuations, $\mathrm{n}(\%)^{\mathrm{b}}$ & $60(51.7)$ & $45(62.5)$ & $15(34.1)$ \\
\hline pRBD, n (\%) & $59(50.8)$ & $36(50)$ & $22(50)$ \\
\hline UPDRS-III score ${ }^{a}$ & $39.6 \pm 18.5$ & $35.8 \pm 16.5$ & $48 \pm 20.8$ \\
\hline Hoehn \& Yahr stage & $2.5 \pm 1.2$ & $2.5 \pm 1.1$ & $2.6 \pm 1.3$ \\
\hline DCFS total score & $12.7 \pm 4.8$ & $13.1 \pm 5.1$ & $12.3 \pm 4.1$ \\
\hline NPI-hallucinations score & $2.3 \pm 3.1$ & $2.5 \pm 3.3$ & $2.1 \pm 2.6$ \\
\hline NPI-total score & $22.3 \pm 18.4$ & $24.8 \pm 20$ & $19.2 \pm 14.9$ \\
\hline NPI-4 score & $9.5 \pm 8.9$ & $10.7 \pm 10.1$ & $7.7 \pm 6.4$ \\
\hline NPI-sleep score & $3.1 \pm 3.9$ & $3.5 \pm 4.1$ & $2.5 \pm 3.5$ \\
\hline DDE, mg ${ }^{b}$ & $317 \pm 295$ & $146 \pm 209$ & $484 \pm 270$ \\
\hline Chl use, n (\%) & $72.4(84)$ & 55 (76.3) & $29(65.9)$ \\
\hline Antipsychotic use, n (\%) & $13(11.2)$ & $11(15.2)$ & $2(4.5)$ \\
\hline \multicolumn{4}{|c|}{$\begin{array}{l}\text { Abbreviations: ChI = cholinesterase inhibitor; DCFS = Dementia Cognitive Fluctuation } \\
\text { bodies; MMSE = Mini-Mental State Examination; MoCA = Montreal Cognitive Asse } \\
\text { dementia; pRBD = probable rapid eye movement sleep behavior disorder; UPDRS-II } \\
\text { Values displayed as mean } \pm \text { SD and percentage (number). } \\
\text { Significant difference between DLB and PDD ( } 2 \text {-tailed independent-samples } t \text { test): } \\
\text { a } p<0.01 \\
\text { b } p<0.001 \text {. }\end{array}$} \\
\hline
\end{tabular}

models. Model fitting was achieved with the maximum likelihood estimation method. Interaction terms between time and intervention and between time and diagnosis were included as fixed effects in initial models for each outcome variable and removed from the final model when they did not achieve significance. On the basis of an a priori statistical plan to account for confounders/covariates, the main-effects (noninteraction) terms were retained in the final models because they were presumed to be still relevant to the parameter estimates of interest (even if not statistically significant). Exploratory models were simplified only when a significant fixed effect was detected (exploratory and final models included in eAppendix 1). All supplementary materials, including eAppendix 1 , eAppendix 2 , and eFigure 1 , are available from Dryad (doi.org/10.5061/dryad.9zw3r22dz).

\section{Data Availability}

Deidentified participant data are available to investigators with appropriate data transfer agreements and institutional board approval.

\section{Results}

\section{Baseline Characteristics}

Group characteristics (DLB vs PDD) are provided in Table 1. There were no significant differences in age, sex, and proportion of patients in the intervention arm between diagnostic groups. However, as expected, patients with PDD had higher severity of motor parkinsonism as scored on UPDRS-III and were taking higher doses of dopaminergic medications.

\section{Cognitive Decline}

Within the time frame of our study, longitudinal cognitive decline was significantly detected with the MMSE (Figure 2 and Table 2). Covariates included age, cholinesterase use, intervention allocation, and diagnosis (PDD or DLB). The group decline of MMSE score over 6 months was -1.3 points (95\% confidence interval $[\mathrm{CI}]-2.2,-0.5 ; p=0.002)$ with an annualized rate of change of -2.7 points (95\% CI $-4.3,-1.0$ ). MoCA scores also showed a strong trend toward a decline with time, although this was not statistically significant $(p=0.06)$. 

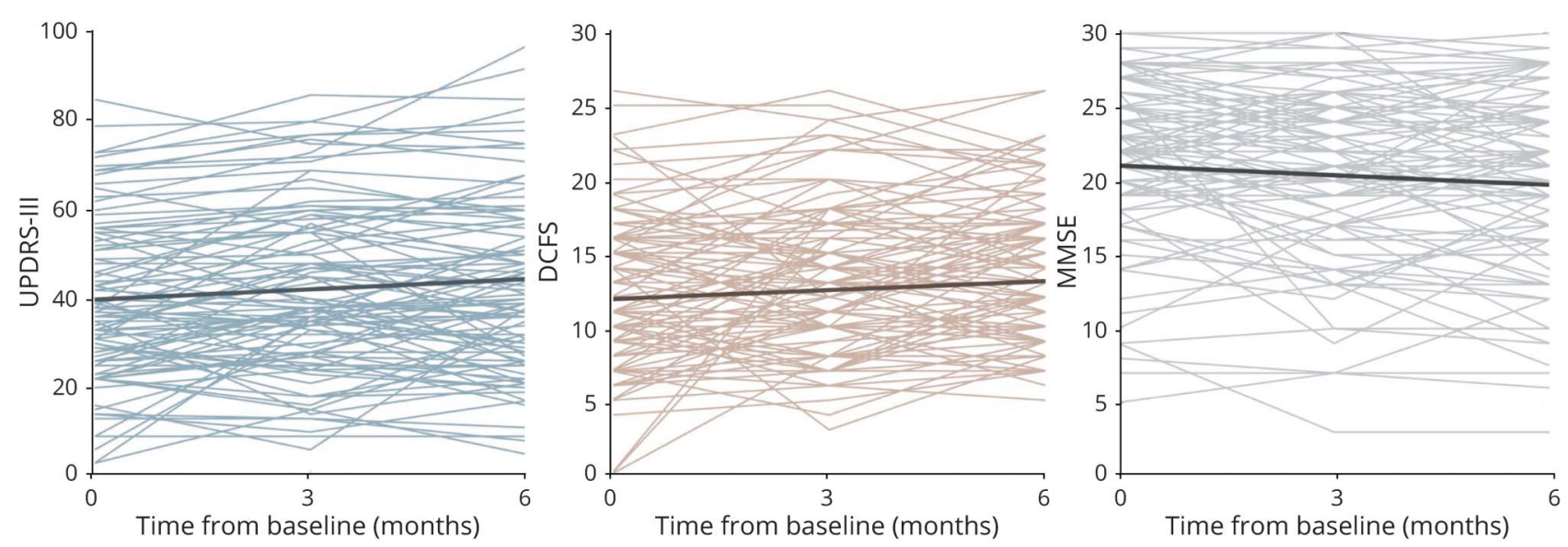

Group-level linear mixed models of fixed effects overlaid onto data for variables in which a significant change in time was detected. Linear trajectory (bold line) is displayed as a function only of time in months from baseline. Mean group variables have been inputted in the model for the other fixed effects within each model (e.g., age, dopaminergic dose). Unified Parkinson's Disease Rating Scale Section III (UPDRS-III) and Dementia Cognitive Fluctuation Scale (DCFS) scores increased significantly over the 6 months of the study, while Mini-Mental State Examination Score (MMSE) score showed a significant decline.

\section{Parkinsonism}

Using a linear mixed model including diagnosis, intervention, age, and levodopa equivalent dose (UPDRS performed in "on" state), we were able to detect a significant change in time in UPDRS-III score across the sample (Figure 1 and Table 2). The estimated rate of change in UPDRS-III score was 3.2 points over 6 months $(95 \%$ CI $0.6,5.9 ; p=0.018)$, with a corresponding annualized change of 6.4 points (95\% CI 1.1, 11.7). There was no significant interaction between presence or absence of Parkinsonism at baseline and change in Parkinsonism over time (eAppendix 2 [doi.org/10.5061/dryad. 9zw3r22dz]).

\section{Cognitive Fluctuations}

A significant increase in DCFS with time was detected over the course of the study (Figure 1 and Table 2) with the use of linear models that covaried for diagnosis, intervention, and cholinesterase use $(p=0.001)$. The 6-month increase on the DCFS scale was estimated to be 1.3 points $(95 \%$ CI $0.6,2.1$ ) with an annualized rate of change of 2.6 points (95\% CI 1.1, 4.1). We did not find a significant effect of any other variables included in the model. There was no significant interaction between presence or absence of cognitive fluctuations at baseline and change in time of the DCFS score (e-appendix 2 [doi.org/10.5061/dryad.9zw3r22dz]).

\section{Neuropsychiatric Features}

Linear mixed models for 3 measures of neuropsychiatric symptoms (NPI-total, NPI-hallucinations score, and NPI-4) were constructed considering intervention, diagnosis, use of cholinesterase inhibitors, use of antipsychotics, and age as covariates (eAppendix 1 [doi.org/10.5061/dryad.9zw3r22dz]). A small proportion of individuals $(\mathrm{n}=10)$ were prescribed additional antipsychotic medications throughout the course of the study. No significant change in time was detected for any of the 3 neuropsychiatric symptom measures (Table 2). Plots of the individual patient scores revealed significant intraindividual variability (eFigure 1 [doi.org/10.5061/dryad.9zw3r22dz]). There was a trend toward reduced NPI-hallucination severity score ( $p=$ 0.06 ) over the time course of this study. However, the small magnitude of change and CIs crossing zero suggests that this was unlikely to be clinically meaningful. There was no significant interaction between the presence or absence of hallucinations at baseline and any of the neuropsychiatric measures (eAppendix 2 [doi.org/10.5061/dryad.9zw3r22dz]).

\section{Sleep Disturbance}

In our initial model, we were unable to detect any significant change in NPI-rated severity of sleep disturbance over the course of this study (Table 2). Covariates included age, diagnosis, intervention, antipsychotic use (sedating actions), and use of cholinesterase inhibitors (adverse reactions with insomnia in some patients). In a post hoc analysis exploring a potential interaction between the presence of sleep disturbance at baseline and change in sleep scores, we found a significant group-level change in sleep disturbance severity over time (0.2 points per month; 95\% CI 0.34, 0.76; $p=0.04$, eAppendix 2 [doi.org/10.5061/dryad.9zw3r22dz]). Furthermore, the presence of sleep disturbance at baseline (NPIsleep score $>0, \mathrm{n}=57$ ) conferred an extra 0.55 -point worsening per month (95\% CI 0.34, 0.76) compared to those without sleep disturbance at baseline.

\section{Individual Variability in Trajectory of Measures (Random Effects)}

Interparticipant variation in the baseline and longitudinal trajectory of the core features was represented through the inclusion of intercept and time (grouped by individuals) as random effects in the models above. Variance estimates of the random effects for each core feature expressed in SDs are 
Table 2 Longitudinal Rate of Change of Key Clinical Features

\begin{tabular}{|c|c|c|c|c|}
\hline \multirow[b]{2}{*}{ Clinical features/measures } & \multicolumn{2}{|c|}{ Time parameter estimates } & \multirow{2}{*}{$\begin{array}{l}\text { Change in score at } 6 \text { mo } \\
(95 \% \mathrm{Cl})\end{array}$} & \multirow{2}{*}{$\begin{array}{l}\text { Annualized (12 mo) rate of change in score } \\
(95 \% \mathrm{Cl})\end{array}$} \\
\hline & Coefficient $(95 \% \mathrm{Cl})$ & $p$ Value & & \\
\hline \multicolumn{5}{|l|}{ Cognitive decline } \\
\hline MMSE score (of 30) & $-0.2(-0.4,-0.1)^{\mathrm{a}}$ & $0.002^{\mathrm{a}}$ & $-1.3(-2.2,-0.5)^{\mathrm{a}}$ & $-2.7(-4.3,-1.0)^{\mathrm{a}}$ \\
\hline MoCA score (of 30) & $-0.1(-0.3,0.01)$ & 0.06 & - & - \\
\hline \multicolumn{5}{|l|}{ Parkinsonism } \\
\hline UPDRS-III score (of 108) & $0.5(0.1,1.0)^{a}$ & $0.018^{\mathrm{a}}$ & $3.2(0.6,5.9)^{\mathrm{a}}$ & $6.4(1.1,11.7)^{a}$ \\
\hline \multicolumn{5}{|l|}{ Cognitive fluctuations } \\
\hline DCFS score (of 20) & $0.2(-0.1,0.3)^{\mathrm{a}}$ & $0.001^{a}$ & $1.3(0.6,2.1)^{\mathrm{a}}$ & $2.6(1.1,4.1)^{\mathrm{a}}$ \\
\hline \multicolumn{5}{|l|}{ Neuropsychiatric symptoms } \\
\hline NPI-hallucinations score (of 12) & $-0.1(-0.3,0.01)$ & 0.06 & - & - \\
\hline NPI-total score (of 180) & $-0.4(-1.0,0.3)$ & 0.29 & - & - \\
\hline NPI-4 score (of 60) & $-0.1(-0.4,0.1)$ & 0.27 & - & - \\
\hline \multicolumn{5}{|l|}{ Sleep disturbance } \\
\hline NPI-sleep score (of 12) & $-0.02(-0.2,0.2)$ & 0.43 & - & - \\
\hline \multicolumn{5}{|c|}{$\begin{array}{l}\text { Abbreviations: } \mathrm{Cl}=\text { confidence interval; DCFS = Dementia Cognitive Fluctuation Scale; MMSE = Mini-Mental State Examination; MoCA = Montreal Cognitive } \\
\text { Assessment; NPI = Neuropsychiatric Inventory; UPDRS-III = Unified Parkinson's Disease Rating Scale Section III. } \\
\text { Coefficient relates to time measured in months from baseline }(0 \text { months). Lower and upper bounds of } 95 \% \text { Cls are provided. } \\
\text { a Extrapolated 6- and 12-month rates of change stated only for variables demonstrating significant change in time }(p<0.05) .\end{array}$} \\
\hline
\end{tabular}

shown in Table 3. Relative to the magnitude of the group-level baseline measures (Table 1) and time fixed-effect estimates (Table 2), large interindividual variation in baseline scores was seen across all measures. Significant interindividual variation in the trajectory of features was also noted for measures of cognitive decline and parkinsonism.

\section{Effect of Diagnosis}

Overall, no significant difference was detected between patients with DLB and those with PDD on the rate of progression of core clinical features in any of the mixed models. However, it is worth noting that despite the lack of significance, the estimates of the effect of diagnosis as a fixed effect did tend to shift the linear trajectory in an expected direction (e.g., higher UPDRS-III score in PDD; higher DCFS score in DLB; see supplementary materials [doi.org/10.5061/dryad. $9 \mathrm{zw} 3 \mathrm{r} 22 \mathrm{dz}])$.

\section{Discussion}

The ability to detect and quantify longitudinal change of key clinical features of Lewy body dementia is essential for understanding the natural history of this disorder and the planning of therapeutic trials. Using common clinical instruments, we have been able to explicitly characterize and quantify the trajectory of several core features in a large cohort of patients with DLB and PDD. Specifically, over the time course of only 6 months, we found it possible to detect significant worsening of motor parkinsonism, cognitive fluctuations, and cognitive decline but not neuropsychiatric symptoms. Furthermore, we were able to quantify the high degree of interindividual variability in clinical trajectories between patients while also showing that, within the time frame of the study, trajectories of these clinical features between PDD and DLB are comparable.

In our study, we were able to detect a significant longitudinal decline using the MMSE. Our annualized rate of decline in MMSE score is consistent with a recent international multicenter cohort study consisting of $>1,000$ patients reporting a mean annual decline of 2.1 points in DLB and 1.8 points in PDD using 3 annual measurements. ${ }^{11}$ A high degree of interparticipant variability has been noted in rate of MMSE score decline in patients with DLB compared with patients with Alzheimer disease $(\mathrm{AD})^{9,28}$ and is consistent with the random effects reported in Table 3 showing an SD of 0.4 points per month around the group-level estimate of -0.2 points per month. As discussed below, significant variability was also seen with the MoCA. This inherent variability may reflect the vulnerability of neuropsychometric measures to the fluctuating cognition characteristic of Lewy body dementia patients (discussed further below). It also highlights the challenge of translating trial methodologies used in other dementias, which are often biased solely toward cognitive outcomes, to Lewy body dementias. ${ }^{8}$ However, previous longitudinal studies in Lewy body dementia emphasize a faster but more variable annual rate of decline in MMSE 
Table 3 Variance Estimates Representing Individual Variation in Baseline and Trajectory of Core Variables

\begin{tabular}{llll}
\hline & Baseline (Intercept) & Time (Slope)/Month & Residual \\
\hline MMSE score & $5.3(4.6,6.1)$ & $0.4(0.2,0.7)$ & $2.5(2.2,2.8)$ \\
\hline MoCA score & $5.3(4.5,6.1)$ & $0.4(0.3,0.6)$ & $2.1(1.8,2.4)$ \\
\hline UPDRS score & $15.3(13.2,17.8)$ & $1.3(0.8,2.1)$ & $7.5(6.5,8.7)$ \\
\hline DCFS score & $4(3.4,4.7)$ & - & $2.5(2.2,2.9)$ \\
\hline NPI-hallucinations score & $1.8(1.5,2.3)$ & - & $2.1(1.9,2.3)$ \\
\hline NPI-total score & $13.2(11.1,15.7)$ & $1.4(0.9,2.3)$ & $9.7(8.5,11.1)$ \\
\hline NPI-4 score & $5.5(4.6,6.6)$ & $0.8(0.5,1.1)$ & $4.4(3.9,5)$ \\
\hline NPI-sleep score & $2.2(1.8,2.8)$ & - & $2.7(2.4,3)$ \\
\hline
\end{tabular}

Abbreviations: DCFS = Dementia Cognitive Fluctuation Scale; MMSE = Mini-Mental State Examination; MoCA = Montreal Cognitive Assessment; NPI = Neuropsychiatric Inventory; UPDRS-III = Unified Parkinson's Disease Rating Scale Section III.

Variance estimates for distribution of intercept, slope, and residuals expressed as SDs (95\% confidence intervals). Random-effect variance estimates were omitted if found to have a nonsignificant effect in the model (Wald test).

scores compared to $\mathrm{AD} .^{9-11,14,15}$ In line with this, our data demonstrate that, despite this variability, MMSE may be a feasible measure of cognitive decline even over a relatively short time frame, demonstrating significant group-level changes over a period of 6 months using 3 time points.

Although the MoCA has been shown to be more sensitive in detecting cognitive impairment in patients with Lewy body dementia, ${ }^{29}$ our results suggest that this tool may be less suited as a measure of change for longitudinal tracking of cognition in trials. As seen here, MoCA scores tend to be consistently lower than MMSE scores, which may be associated with floor effects in the evaluation of changes over time. Furthermore, MoCA scores have been shown to be associated with higher individual variability at baseline compared to MMSE scores in patients with Lewy body dementia. ${ }^{10}$ Thus, while MoCA has significant diagnostic value, its prognostic limitations may restrict its utility as a marker of cognitive decline in longitudinal trials.

The UPDRS is the most frequently used outcome measure in symptomatic trials of patients with Parkinson disease. ${ }^{30}$ More recently, with the emergence of disease-modifying trials, there is also growing use of the UPDRS motor score as an endpoint to track disease progression. ${ }^{31}$ We found that, even considering levodopa use and baseline variation in scores, a significant change in motor parkinsonism rated with the UPDRS-III could be estimated, corresponding to an increase of 3.2 points over 6 months and an annualized rate of change of 6.4 points. These values are comparable to the previously determined minimal clinically important change of 5 points on the UPDRS motor in trials of Parkinson disease. ${ }^{32,33}$ Furthermore, the increase in UPDRS-III score at 6 months in Lewy body dementia exceeds the annual rate of change of 2.4 points per year reported in early untreated Parkinson disease. ${ }^{34}$ While the minimal clinically important change has yet to be specifically defined for Lewy body dementia, the comparatively rapid rate of change reported in our study supports parkinsonism as a feasible outcome measure for clinical trials assessing the impact of diseasemodifying therapies in Lewy body dementias.

In this study, we were able to systematically investigate the progression of cognitive fluctuations. We found a significant group-level increase of 1.3 points in DCFS score over 6 months, with an annualized rate of change of 2.6 points over 12 months. The DCFS is a clinically validated rating scale demonstrating good sensitivity, specificity, and test-retest and interrater reliabilities. ${ }^{23}$ The present version of the scale is based on the summed responses of items from the original scale found to best discriminate between patients with and without cognitive fluctuations (i.e., marked differences in functioning during the daytime, daytime somnolence, daytime drowsiness, and altered levels of consciousness during the day). ${ }^{23} \mathrm{~A}$ minimal difference in score constituting a clinically meaningful change to patients and their caregivers has yet to be determined for the DCFS. The modest size differences at the group level may relate to the psychometric properties of the scale itself. It may also be accounted for by the considerable variability in the expression of this symptom between patients, noting some patients did not report symptoms of cognitive fluctuations during the study (Figure 1). Furthermore, the lack of significance of including time as a random effect in our cohort suggests that there was less interindividual variability in the progression of this marker and that fluctuation symptoms may track more uniformly between patients than other clinical measures. Cognitive fluctuations have recently been reported to occur in high frequency in the mild cognitive impairment stage of DLB. ${ }^{35}$ Thus, as we move toward earlier diagnosis and recruitment of patients into disease-modifying trials, our findings highlight the potential utility of fluctuation severity as an outcome measure even in prodromal populations.

We were unable to detect any significant progression in hallucinations and other composite measures of psychiatric symptoms using the NPI over the time course of our study. 
Neuropsychiatric manifestations across all dementias are highly variable in their prevalence and expression. ${ }^{36}$ In Lewy body dementias, psychiatric symptoms such as visual hallucinations are present in up to $80 \%$ of patients and regarded as a core diagnostic feature for the disorder, ${ }^{2}$ and the absence of quantifiable progression needs to be reconciled with the accepted impact of neuropsychiatric symptoms on quality of life, caregiver burden, and risk of institutionalization. ${ }^{37,38}$ It is likely that many neuropsychiatric features reach a certain level of prominence before diagnosis with only modest further deterioration detected in the time course of this study. This is reflected by high baseline measures of NPI symptoms (Table 1) and is consistent with a recent study of patients with mild dementia that found a high proportion of reported neuropsychiatric symptoms at baseline and only modest changes in absolute NPI scores over 5 years. ${ }^{39}$ Alternatively, the lack of a significant change in continuous measures of hallucinations may suggest that categorical variables (e.g., absence or presence) might be a more appropriate means of capturing symptom change over time. In line with this, a recent longitudinal study comparing categorical state changes of neuropsychiatric symptoms between $\mathrm{DLB}$ and $\mathrm{AD}$ over a 12 -year period found that patients with DLB were more likely to exhibit a relapsing/remitting nature rather than continuous worsening or improvement. ${ }^{17}$ Currently, there is no scale designed specifically for measuring visual hallucinations in DLB, and our results highlight the need to develop such a scale or instead consider testing alternative scales validated in other populations such as the Psychosis and Hallucinations Questionnaire ${ }^{40}$ or the North-East Visual Hallucinations Interview. ${ }^{41}$

$\mathrm{RBD}$ is a core diagnostic feature of $\mathrm{DLB},{ }^{2}$ and sleep disturbances more generally are increasingly recognized as an important symptom complex of Lewy body disorders. ${ }^{42,43}$ The longitudinal progression of sleep disturbances in Lewy body dementias has not been explicitly investigated. In our study, the NPI-sleep was used as a pragmatic caregiver and patientcentered measure of overall burden of sleep disturbances, including, but not limited to, disruptive dream enactment behaviors. We found that there was a significant change in the burden of sleep disturbance over the 6-month time frame, which was present only once the interaction between the presence of sleep disturbance and time was taken into account. This finding suggests that there is a detectable worsening of sleep disturbances over time in DLB and PDD. Further prospective studies using questionnaires targeting various aspects of sleep (e.g., insomnia, fragmentation, nocturia) and assessment of RBD specifically (such as through the use of sleep diaries ${ }^{44}$ ) will be required to understand the trajectory of this symptom complex and its feasibility as an endpoint in symptomatic and diseasemodifying clinical trials in Lewy body dementia.

DLB and PDD share many clinical, neurochemical, and pathologic features, distinguished by the timing of onset of cognitive symptoms relative to parkinsonism. ${ }^{45,46}$ Such distinction results in the differential expression of core symptoms at baseline (Table 1). However, PDD and DLB were not significant covariates in the group-level trajectory of any core symptom and did not interact with time. This is aligned with previous studies finding no significant difference between the groups in relation to cognitive decline, ${ }^{47}$ time to nursing home admission, ${ }^{14}$ and survival. ${ }^{48}$ Thus, we conclude that over time courses comparable to that of this study, the trajectory of core features between the 2 disorders can be expected to be similar, which (depending on the nature of the intervention) supports the practice of combining the 2 groups to improve power in studies in which investigators wish to use change in core features as endpoints.

Although average trajectories of symptoms are useful for guiding group-level endpoints, the high interparticipant variability demonstrates the inherent heterogeneity of patients with Lewy body dementia. As a result, such trajectories cannot be applied at the individual level in clinical practice. Furthermore, although the aim here was to use more common and validated tools for exploring such symptoms that are likely to be used as endpoints in clinical trials, the use of composite endpoints (such as NPI-sleep) inherently compromises sensitivity to more specific features of a symptom complex (e.g., daytime somnolence), which may on their own be a marker of progression. Studies exploring more isolated symptoms with richer rating scales designed for such symptoms (e.g., use of Epworth Sleepiness Scale ${ }^{49}$ for daytime somnolence) may be necessary to properly understand the trajectory of individual symptoms. Likewise, other endpoints not used here such as detailed neuropsychological testing (recently found to differentiate PDD and DLB ${ }^{16}$ ) may also be useful adjuncts for measuring clinical progression across specific symptom domains. Several endpoints were informed by a close informant (mainly partners and close family members), but whether specific features of the informants/caregivers (such as demographics) affect reporting should be considered in future studies. Cognitive endpoints using tests such as the MMSE have been used commonly in many recent disease-modifying trials in $\mathrm{AD}$ and have been the focus of most longitudinal studies of the natural history of Lewy body dementia to date. ${ }^{9,11,14}$ However, cognitive measures are dependent on a patient's state on the day of testing and are especially affected by fluctuations in patients with Lewy body dementia. ${ }^{50}$ This was confirmed in our study by the statistically significant degree of individual variability. This argues in favor of the use of semiquantitative instruments (such as the DCFS) that ask the patient and informant to comment on the last month and are therefore less likely to be confounded by such state-dependent variations.

That progression of certain features could not be detected may be a function of the limited duration of the study. However, the relatively rapid progression of this disease is an important consideration, and such a time frame was felt important to accommodate enrollment of individuals with DLB with a diverse range of severity while also ensuring a high rate of completion as seen in our study. Furthermore, the use of a linear mixed modeling approach, which assesses the trajectory and variability of a disease measure, offers potentially greater 
power for detecting significant differences in symptom progression over time compared to the more traditionally used method in clinical trials of assessing only the absolute difference between baseline and a single point in time at the end of the study. This latter point is especially pertinent to patients with Lewy body dementia who display significant degrees of interindividual and intraindividual variability. Such considerations will also be relevant in future studies, especially clinical trials, in which duration and sample size are often also dictated by funding constraints. Indeed, power and sample size calculations are the necessary next step to operationalize our findings. However, outstanding questions remain regarding the minimal clinically important differences for fluctuation and sleep-related measures, as well as the appropriate study design (e.g., comparing absolute change at 6 months and change in trajectories using linear mixed modeling approaches). Future studies involving validation of our results in larger samples, use of caregiver-reported scales to support minimal clinically important differences, and power calculations derived with simulation-based approaches will be essential to facilitate planning of clinical trials in DLB.

Last, as with most clinical trials, demographic data were not collected for those who declined participation (38\%). Years of education was also not captured in this study. Care should be taken when generalizing to patients in other countries with potentially different practice patterns and educational, racial, and ethnic backgrounds.

Lewy body dementias are heterogeneous disorders, and defining disease progression through clinical measures remains an urgent challenge for the field. The detection and quantification of a significant change in the trajectory of key clinical features and symptoms over a relatively short time frame, beyond just cognitive decline, suggest the feasibility of using some of these features as clinical endpoints in isolation or as part of a composite measure in therapeutic trials. These findings also reinforce the Lewy body dementias as a tractable disease model for testing disease-modifying interventions.

\section{Study Funding}

This article summarizes independent research funded by the National Institute for Health Research (NIHR) under its Programme Grants for Applied Research Programme (grant reference No. DTC-RP-PG-0311-12001) and supported by NIHR Newcastle Biomedical Research Centre based at Newcastle Upon Tyne Hospitals NHS Foundation Trust and Newcastle University.

\section{Disclosure}

E. Matar was funded by the Endeavour Leadership Program (Australian Department of Education, Skills and Employment) and a National Health and Medical Research Council (NHMRC) postgraduate scholarship (No. 1151722). E. Matar is employed by New South Wales Health and has provided Seed Investment to Elly Health. G.M. Halliday is an NHMRC Leadership Fellow. G.M. Halliday has done paid committee work for the NHMRC, Australia and served on advisory boards of the Danish Research Institute of Translational Neuroscience, Aarhus University, and Queensland Institute for Medical Research, Australia. She has received royalties from Academic Press, Elsevier, \& Oxford University Press. S.J.G. Lewis has received grant funding from the NHMRC and Parkinson's Foundation Australia and is employed by the University of Sydney. S.J.G. Lewis has received honoraria from the International Movement Disorders Society. S.R. White was funded by the NIHR Cambridge Biomedical Research Centre. A. Thomas, S.R. White, I.G. McKeith, J.-P. Taylor, A. Surendranathan, J.P.M. Kane, and J.T. O'Brien have received grant funding from the NIHR and have no additional financial disclosures. Go to Neurology. org/ $\mathrm{N}$ for full disclosures.

\section{Publication History}

Received by Neurology January 11, 2021. Accepted in final form June 15, 2021.

Appendix Authors

\begin{tabular}{|c|c|c|}
\hline Name & Location & Contribution \\
\hline Elie Matar, MD & $\begin{array}{l}\text { Department of Psychiatry, } \\
\text { University Cambridge, UK; } \\
\text { Forefront Parkinson's } \\
\text { Disease Research Clinic, } \\
\text { University of Sydney, } \\
\text { Australia; Brain and Mind } \\
\text { Centre, Faculty of } \\
\text { Medicine and Health, } \\
\text { University of Sydney, } \\
\text { Australia }\end{array}$ & $\begin{array}{l}\text { Responsible for } \\
\text { conception, design, and } \\
\text { execution of the research } \\
\text { project and statistical } \\
\text { analysis, as well as writing } \\
\text { of the first draft }\end{array}$ \\
\hline
\end{tabular}

\begin{tabular}{lll}
\hline Simon R. White, & Department of Psychiatry, & Responsible for design, \\
PhD & University Cambridge, UK; & review, and critique of the \\
& MRC Biostatistics Unit, & statistical analysis and \\
& University of Cambridge, & review and critique of the \\
& UK & manuscript
\end{tabular}

John-Paul Taylor, Newcastle Translational Involved in the design and MD, PhD and Clinical Research execution of the research Institute, Campus for project, review and Ageing and Vitality, critique of the manuscript, Newcastle University, and statistical analysis Newcastle Upon Tyne, UK

\begin{tabular}{lll}
\hline Alan Thomas, & Newcastle Translational & Involved in the design, \\
MD, PhD & and Clinical Research & $\begin{array}{l}\text { execution of the research } \\
\text { project, review and }\end{array}$ \\
& Institute, Campus for & $\begin{array}{l}\text { Ageing and Vitality, } \\
\text { critique of the manuscript, } \\
\text { Newcastle University, }\end{array}$ \\
& and statistical analysis \\
& & \\
\hline
\end{tabular}

Ian G. McKeith, Newcastle Translational Involved in the design and MD, FMedSci and Clinical Research execution of the research Institute, Campus for project, review and Ageing and Vitality, critique of the manuscript, Newcastle University, and statistical analysis Newcastle Upon Tyne, UK

\begin{tabular}{lll}
\hline Joseph P.M. Kane, & $\begin{array}{l}\text { Centre for Public Health, } \\
\text { Queens University Belfast, } \\
\text { PhD }\end{array}$ & $\begin{array}{l}\text { Responsible for execution } \\
\text { of the research project } \\
\text { and review and critique of } \\
\text { the manuscript }\end{array}$ \\
& \\
\hline
\end{tabular}

Ajenthan Department of Psychiatry, Responsible for execution Surendranathan, University Cambridge, UK of the research project MD and review and critique of the manuscript 
Appendix (continued)

\begin{tabular}{|c|c|c|}
\hline Name & Location & Contribution \\
\hline $\begin{array}{l}\text { Glenda M. } \\
\text { Halliday, PhD }\end{array}$ & $\begin{array}{l}\text { Forefront Parkinson's } \\
\text { Disease Research Clinic, } \\
\text { University of Sydney, } \\
\text { Australia; Brain and Mind } \\
\text { Centre, Faculty of } \\
\text { Medicine and Health, } \\
\text { University of Sydney, } \\
\text { Australia }\end{array}$ & $\begin{array}{l}\text { Responsible for } \\
\text { organization of the project } \\
\text { and review and critique of } \\
\text { the manuscript }\end{array}$ \\
\hline $\begin{array}{l}\text { Simon J. G. Lewis, } \\
\text { MD, PhD }\end{array}$ & $\begin{array}{l}\text { Forefront Parkinson's } \\
\text { Disease Research Clinic, } \\
\text { University of Sydney, } \\
\text { Australia; Brain and Mind } \\
\text { Centre, Faculty of } \\
\text { Medicine and Health, } \\
\text { University of Sydney, } \\
\text { Australia }\end{array}$ & $\begin{array}{l}\text { Responsible for } \\
\text { organization of the project } \\
\text { and review and critique of } \\
\text { the manuscript }\end{array}$ \\
\hline $\begin{array}{l}\text { John T. O'Brien, } \\
\text { DM, FRCPsych }\end{array}$ & $\begin{array}{l}\text { Department of Psychiatry, } \\
\text { University Cambridge, UK }\end{array}$ & $\begin{array}{l}\text { Responsible for } \\
\text { conception, execution, } \\
\text { organization, and design } \\
\text { of the study and review } \\
\text { and critique of the } \\
\text { manuscript }\end{array}$ \\
\hline
\end{tabular}

\section{References}

1. Walker Z, Possin KL, Boeve BF, Aarsland D. Lewy body dementias. Lancet. 2015; 386(10004):1683-1697.

2. McKeith IG, Boeve BF, Dickson DW, et al. Diagnosis and management of dementia with Lewy bodies: fourth consensus report of the DLB Consortium. Neurology. 2017; 89(1):88-100.

3. Emre M, Aarsland D, Brown R, et al. Clinical diagnostic criteria for dementia associated with Parkinson's disease. Mov Disord. 2007;22(12):1689-1707. quiz 1837.

4. Zaccai J, McCracken C, Brayne C. A systematic review of prevalence and incidence studies of dementia with Lewy bodies. Age Ageing. 2005;34(6):561-566.

5. Mueller C, Perera G, Rajkumar AP, et al. Hospitalization in people with dementia with Lewy bodies: frequency, duration, and cost implications. Alzheimers Dement (Amst) 2018;10:143-152.

6. Mueller C, Ballard C, Corbett A, Aarsland D. The prognosis of dementia with Lewy bodies. Lancet Neurol. 2017;16(5):390-398.

7. Bostrom F, Jonsson L, Minthon L, Londos E. Patients with dementia with Lewy bodies have more impaired quality of life than patients with Alzheimer disease. Alzheimer Dis Assoc Disord. 2007;21(2):150-154.

8. Goldman JG, Forsberg LK, Boeve BF, et al. Challenges and opportunities for improving the landscape for Lewy body dementia clinical trials. Alzheimers Res Ther. 2020;12(1):137.

9. Rongve A, Soennesyn H, Skogseth R, et al. Cognitive decline in dementia with Lewy bodies: a 5-year prospective cohort study. BMJ Open. 2016;6(2):e010357.

10. Biundo R, Weis L, Bostantjopoulou S, et al. MMSE and MoCA in Parkinson's disease and dementia with Lewy bodies: a multicenter 1-year follow-up study. J Neural Transm (Vienna). 2016;123(4):431-438.

11. Kramberger MG, Auestad B, Garcia-Ptacek S, et al. Long-term cognitive decline in dementia with Lewy bodies in a large multicenter, international cohort. J Alzheimers Dis. 2017;57(3):787-795.

12. Breitve MH, Chwiszczuk LJ, Bronnick K, et al. A longitudinal study of neurocognition in dementia with Lewy bodies compared to Alzheimer's disease. Front Neurol. 2018;9: 124

13. Breitve $\mathrm{MH}$, Hynninen MJ, Bronnick $\mathrm{K}$, et al. A longitudinal study of anxiety and cognitive decline in dementia with Lewy bodies and Alzheimer's disease. Alzheimers Res Ther. 2016;8:3.

14. Williams MM, Xiong C, Morris JC, Galvin JE. Survival and mortality differences between dementia with Lewy bodies vs Alzheimer disease. Neurology. 2006;67(11): 1935-1941.

15. Burn DJ, Rowan EN, Allan LM, Molloy S, O’Brien JT, McKeith IG. Motor subtype and cognitive decline in Parkinson's disease, Parkinson's disease with dementia, and dementia with Lewy bodies. J Neurol Neurosurg Psychiatry. 2006;77(5):585-589.

16. Smirnov DS, Galasko D, Edland SD, Filoteo JV, Hansen LA, Salmon DP. Cognitive decline profiles differ in Parkinson disease dementia and dementia with Lewy bodies. Neurology. 2020;94(20):e2076-e2087.

17. Vik-Mo AO, Giil LM, Borda MG, Ballard C, Aarsland D. The individual course of neuropsychiatric symptoms in people with Alzheimer's and Lewy body dementia: 12year longitudinal cohort study. Br J Psychiatry. 2020;216(1):43-48.

18. Emre M, Tsolaki M, Bonuccelli U, et al. Memantine for patients with Parkinson's disease dementia or dementia with Lewy bodies: a randomised, double-blind, placebocontrolled trial. Lancet Neurol. 2010;9(10):969-977.
19. McKeith I, Del Ser T, Spano P, et al. Efficacy of rivastigmine in dementia with Lewy bodies: a randomised, double-blind, placebo-controlled international study. Lancet. 2000;356(9247):2031-2036

20. O’Brien JT, McKeith IG, Thomas AJ, et al. Introduction of a management toolkit for Lewy body dementia: a pilot cluster-randomized trial. Mov Disord. 2021;36(1):143-151.

21. Taylor JP, McKeith IG, Burn DJ, et al. New evidence on the management of Lewy body dementia. Lancet Neurol. 2020;19(2):157-169.

22. Goetz CG, Tilley BC, Shaftman SR, et al. Movement Disorder Society-sponsored revision of the Unified Parkinson's Disease Rating Scale (MDS-UPDRS): scale presentation and clinimetric testing results. Mov Disord. 2008;23(15):2129-2170.

23. Lee DR, McKeith I, Mosimann U, et al. The Dementia Cognitive Fluctuation Scale, a new psychometric test for clinicians to identify cognitive fluctuations in people with dementia. Am J Geriatr Psychiatry. 2014;22(9):926-935.

24. Cummings JL, Mega M, Gray K, Rosenberg-Thompson S, Carusi DA, Gornbein J. The Neuropsychiatric Inventory: comprehensive assessment of psychopathology in dementia. Neurology. 1994;44(12):2308-2314.

25. Del Ser T, McKeith I, Anand R, Cicin-Sain A, Ferrara R, Spiegel R. Dementia with Lewy bodies: findings from an international multicentre study. Int $J$ Geriatr Psychiatry. 2000;15(11):1034-1045.

26. Mori E, Ikeda M, Kosaka K; Donepezil-DLB Study Investigators. Donepezil for dementia with Lewy bodies: a randomized, placebo-controlled trial. Ann Neurol. 2012;72(1):41-52.

27. Baayen RH, Davidson DJ, Bates DM. Mixed-effects modeling with crossed random effects for subjects and items. J Mem Lang. 2008;59(4):390-412.

28. Giil LM, Aarsland D. Greater variability in cognitive decline in Lewy body dementia compared to Alzheimer's disease. J Alzheimers Dis. 2020;73(4):1321-1330.

29. Matar E, Ehgoetz Martens KA, Halliday GM, Lewis SJG. Clinical features of Lewy body dementia: insights into diagnosis and pathophysiology. J Neurol. 2020;267(2): 380-389.

30. Schrag A, Sampaio C, Counsell N, Poewe W. Minimal clinically important change on the Unified Parkinson's Disease Rating Scale. Mov Disord. 2006;21(8):1200-1207.

31. Venuto CS, Potter NB, Dorsey ER, Kieburtz K. A review of disease progression models of Parkinson's disease and applications in clinical trials. Mov Disord. 2016; 31(7):947-956.

32. Horvath K, Aschermann Z, Acs P, et al. Minimal clinically important difference on the Motor Examination part of MDS-UPDRS. Parkinsonism Relat Disord. 2015;21(12): 1421-1426.

33. Makkos A, Kovacs M, Aschermann Z, et al. Are the MDS-UPDRS-based composite scores clinically applicable?. Mov Disord. 2018;33(5):835-839.

34. Holden SK, Finseth T, Sillau SH, Berman BD. Progression of MDS-UPDRS scores over five years in de novo Parkinson disease from the Parkinson's Progression Markers Initiative Cohort. Mov Disord Clin Pract. 2018;5(1):47-53.

35. Donaghy PC, Taylor JP, O'Brien JT, et al. Neuropsychiatric symptoms and cognitive profile in mild cognitive impairment with Lewy bodies. Psychol Med. 2018;48(14):2384-2390.

36. Cerejeira J, Lagarto L, Mukaetova-Ladinska EB. Behavioral and psychological symptoms of dementia. Front Neurol. 2012;3:73.

37. Lee DR, McKeith I, Mosimann U, Ghosh-Nodyal A, Thomas AJ. Examining carer stress in dementia: the role of subtype diagnosis and neuropsychiatric symptoms. Int Geriatr Psychiatry. 2013;28(2):135-141.

38. Svendsboe E, Terum T, Testad I, et al. Caregiver burden in family carers of people with dementia with Lewy bodies and Alzheimer's disease. Int J Geriatr Psychiatry. 2016;31(9):1075-1083.

39. Vik-Mo AO, Giil LM, Ballard C, Aarsland D. Course of neuropsychiatric symptoms in dementia: 5-year longitudinal study. Int J Geriatr Psychiatry. 2018;33(10):1361-1369.

40. Shine JM, Mills JMZ, Qiu J, et al. Validation of the psychosis and hallucinations questionnaire in non-demented patients with Parkinson's disease. Mov Disord Clin Pract. 2015;2(2):175-181.

41. Mosimann UP, Collerton D, Dudley R, et al. A semi-structured interview to assess visual hallucinations in older people. Int J Geriatr Psychiatry. 2008;23(7):712-718

42. Bugalho P, Salavisa M, Marto JP, Borbinha C, Alves L. Polysomnographic data in dementia with Lewy bodies: correlation with clinical symptoms and comparison with other alpha-synucleinopathies. Sleep Med. 2019;55:62-68.

43. Bliwise DL, Mercaldo ND, Avidan AY, Boeve BF, Greer SA, Kukull WA. Sleep disturbance in dementia with Lewy bodies and Alzheimer's disease: a multicenter analysis. Dement Geriatr Cogn Disord. 2011;31(3):239-246.

44. Gilat M, Coeytaux Jackson A, Marshall NS, et al. Melatonin for rapid eye movement sleep behavior disorder in Parkinson's disease: a randomised controlled trial. Mov Disord. 2020;35(2):344-349.

45. Jellinger KA. Dementia with Lewy bodies and Parkinson's disease-dementia: current concepts and controversies. J Neural Transm (Vienna). 2018;125(4):615-650.

46. Ferman TJ, Aoki N, Boeve BF, et al. Subtypes of dementia with Lewy bodies are associated with $a$-synuclein and tau distribution. Neurology. 2020;95(2):e155-e165.

47. Fereshtehnejad SM, Lokk J, Wimo A, Eriksdotter M. No significant difference in cognitive decline and mortality between Parkinson's disease dementia and dementia with Lewy bodies: naturalistic longitudinal data from the Swedish Dementia Registry. J Parkinsons Dis. 2018;8(4):553-561.

48. Savica R, Grossardt BR, Bower JH, et al. Survival and causes of death among people with clinically diagnosed synucleinopathies with parkinsonism: a population-based study. JAMA Neurol. 2017;74(7):839-846.

49. Johns MW. A new method for measuring daytime sleepiness: the Epworth Sleepiness Scale. Sleep. 1991;14(6):540-545.

50. Matar E, Shine JM, Halliday GM, Lewis SJG. Cognitive fluctuations in Lewy body dementia: towards a pathophysiological framework. Brain. 2020;143(1) 31-46. 


\section{Neurology}

\section{Progression of Clinical Features in Lewy Body Dementia Can Be Detected Over 6 Months}

Elie Matar, Simon R. White, John-Paul Taylor, et al.

Neurology 2021;97; e1031-e1040 Published Online before print August 17, 2021

DOI 10.1212/WNL.0000000000012450

This information is current as of August 17, 2021

\section{Updated Information \&} Services

References

Subspecialty Collections

Permissions \& Licensing

Reprints including high resolution figures, can be found at: http://n.neurology.org/content/97/10/e1031.full

This article cites 50 articles, 7 of which you can access for free at: http://n.neurology.org/content/97/10/e1031.full\#ref-list-1

This article, along with others on similar topics, appears in the following collection(s):

Cohort studies

http://n.neurology.org/cgi/collection/cohort_studies

Dementia with Lewy bodies

http://n.neurology.org/cgi/collection/dementia_with_lewy_bodies

Natural history studies (prognosis)

http://n.neurology.org/cgi/collection/natural_history_studies_prognosis

\section{Parkinson's disease with dementia}

http://n.neurology.org/cgi/collection/parkinsons_disease_with_dementi a

Information about reproducing this article in parts (figures,tables) or in its entirety can be found online at:

http://www.neurology.org/about/about_the_journal\#permissions

Information about ordering reprints can be found online:

http://n.neurology.org/subscribers/advertise

Neurology ${ }^{\circledR}$ is the official journal of the American Academy of Neurology. Published continuously since 1951, it is now a weekly with 48 issues per year. Copyright Copyright ( 2021 The Author(s). Published by Wolters Kluwer Health, Inc. on behalf of the American Academy of Neurology.. All rights reserved. Print ISSN: 0028-3878. Online ISSN: 1526-632X.

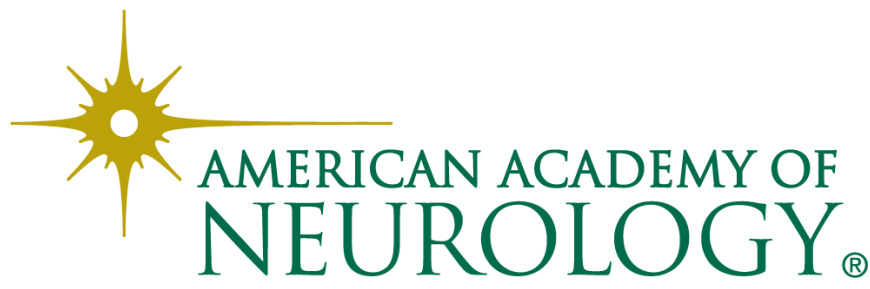

